\title{
Matrice lipidique et biodisponibilité de l'acide alpha-linolénique
}

\author{
Leslie Couëdelo*, Aurélie Termon et Carole Vaysse \\ Université Bordeaux Segalen, 146 rue Léo Saignat, 33076 Bordeaux cedex, France
}

Reçu le 30 novembre 2016 - Accepté le 18 janvier 2017

\begin{abstract}
Résumé - Les acides gras poly-insaturés (AGPI) de la série oméga-3 ( $\omega 3$ ) ont un rôle important dans la prévention de certaines pathologies. En plus d'être nécessaires dans des conditions physiologiques particulières (développement pré- et post-natal, croissance) (Riediger et al., 2009. J Am Diet Assoc 109 : 668-679), ils sont associés à des effets santé en termes de prévention, notamment au niveau de pathologies cardiovasculaires, inflammatoires, certains cancers et certaines maladies neuro-dégénératives (De Lorgeril et al., 1994. Lancet 343 : 1454-1459 ; Simopoulos, 2008. Exp Biol Med (Maywood, NJ) 233 : 674-688). Cependant, les dernières études épidémiologiques montrent que les apports en AGPI $\omega 3$, et notamment en acide alpha-linolénique (ALA), précurseur métabolique des AGPI à longue chaîne $\omega 3$, sont deux fois inférieurs aux recommandations de l'Agence national de sécurité sanitaire, de l'alimentation, de l'environnement et du travail (ANSES, 2011). Outre la nécessité d'augmenter l'apport en ALA, il est désormais nécessaire de prendre en considération les facteurs qui améliorent sa biodisponibilité. Dans ce contexte, nous avons testé plusieurs paramètres susceptibles de moduler le devenir métabolique de l'ALA. Nos recherches ont mis en évidence que plusieurs paramètres physiques et chimiques, tels que l'émulsification d'une huile linolénique avec de la lécithine de soja, la position de l'ALA sur le squelette glycérique du triglycéride alimentaire mais aussi la composition de la matrice permettraient de moduler la biodisponibilité et le devenir métabolique de l'ALA dans l'organisme.
\end{abstract}

Mots clés : alpha-linolénique / biodisponibilité / émulsion / structure glycéridique / matrice lipidique

\begin{abstract}
Lipid matrix and bioavailability of alpha-linolenic acid. Polyunsaturated fatty acids (PUFA) of omega-3 series ( $\omega 3)$ have an important role in preventing certain diseases. Besides being necessary in specific physiological conditions (pre- and post-natal development, growth) (Riediger et al., 2009.J Am Diet Assoc 109: 668-679.), they are associated with health effects in terms of prevention, especially for cardiovascular disease, inflammation, some cancers and neurodegenerative diseases (De Lorgeril et al., 1994. Lancet 343: 1454-1459; Simopoulos, 2008. Exp Biol Med (Maywood, NJ) 233: 674-688). However, recent epidemiological studies show that the PUFA $\omega 3$ intake, including alpha-linolenic acid (ALA), the metabolic precursor of long chain PUFA $\omega 3$, are three times lower than the French recommendations (ANSES, 2011). Besides the need to increase the ALA intake, it is now necessary to take into account the factors that improve its bioavailability. In this context, we tested several parameters that modulate the metabolic fate of ALA. Our research has demonstrated that several physical and chemical parameters, such as the emulsification of linolenic oil with soybean lecithin, the position of ALA on the glycerol backbone of the triglyceride but also the composition of the lipid matrix would modulate the bioavailability and the metabolic fate of ALA in the organism.
\end{abstract}

Keywords: alpha linolenic acid / bioavailability / emulsion / triglyceride structure / lipid matrix

\section{Introduction}

L'acide alpha-linolénique (ALA ; C18:3 n-3) est le précurseur des acides gras poly-insaturés à longues chaînes oméga-3 (AGPI-LC $\omega 3$ ), i.e. l'acide eicosapentaénoïque (EPA ; 20:5 n-3), l'acide docosapentaénoïque (DPA ;

\footnotetext{
*Correspondance : 1.couedelo@iterg.com
}

22:5 n-3) et l'acide docosahexaénoïque (DHA ; 22:6 n-3). L'ALA est associé à une diminution du risque cardiovasculaire $(\mathrm{RCV})$ à travers divers mécanismes biologiques, notamment la fonction plaquettaire, l'inflammation, la fonction des cellules endothéliales, la compliance artérielle et l'arythmie (De Lorgeril et al., 1994 ; Hu et al., 1999 ; Bemelmans et al., 2002 ; Zhao et al., 2004). Toutefois, les études s'accordent à dire que l'ALA serait un excellent substrat pour la $\beta$-oxydation et qu'une majeure partie serait 


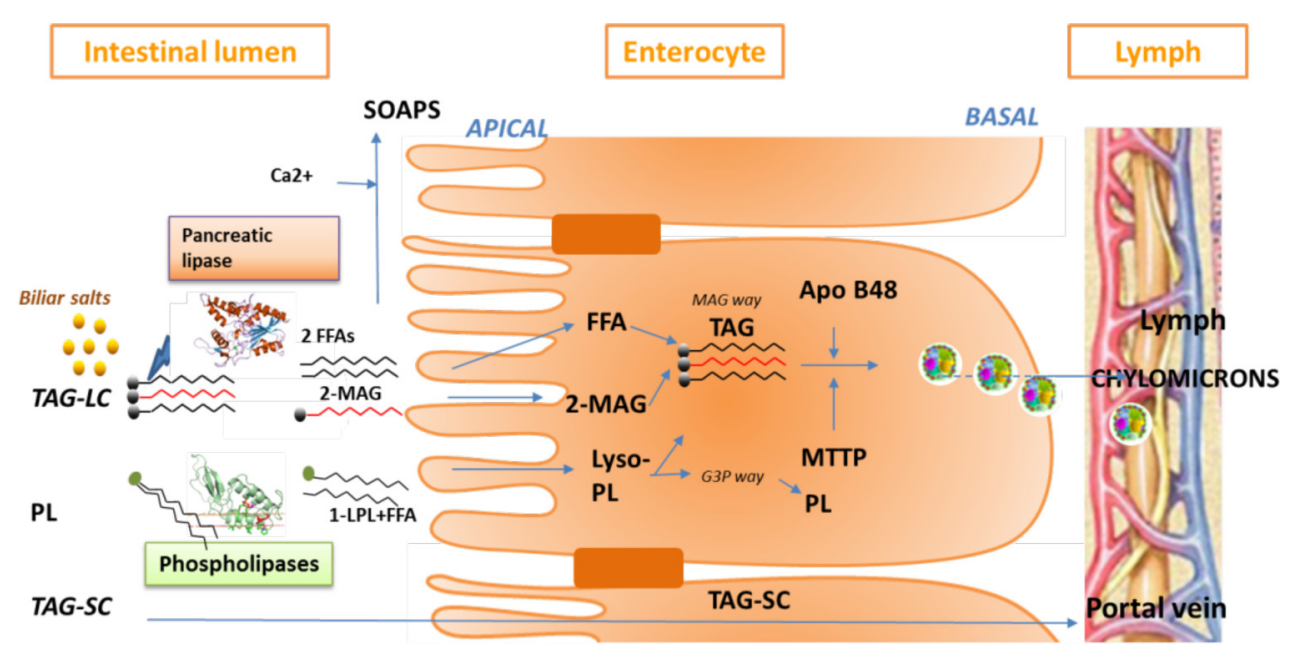

Fig. 1. Schéma des voies de biosynthèse des triglycérides (triacylglycerol [TAG]) dans l'entérocyte. La voie principale de synthèse des monoglycérides (MAG pathway) forme des TAG à partir des 2-MAG et des acides gras libres (FFA) après deux acylations successives par la monoacylglycérol : acylCoA acyltransférase (MGAT) et les diacylglycérol : acylCoA acyltransférases 1 et 2 (DGAT1 et DGAT2) dans le réticulum endoplasmique. La voie secondaire de synthèse des triglycérides (glycerol phosphate pathway; G3P way) passe par l'acide phosphatidique formé à partir du glycérol-3-phosphate, sous l'action de deux acylations successives par les glycérol-3-phosphate acyltransférases, suivie d'une déphosphorylation en diacylglycérol par la phosphatidate phosphohydrolase et d'une troisième acylation par les DGAT1 et DGAT2 ; cette dernière étape est commune aux deux voies de biosynthèse des TAG.

$\beta$-oxydée (15 à $33 \%$ ) (Burdge et Wootton, 2002 ; Burdge, 2004 ; Arterburn et al., 2006). Seule une faible proportion serait convertie en EPA ( 8 à $21 \%$ ) et en DHA (0,05 à $9 \%$ ) (Burdge et Wootton, 2002 ; Burdge, 2004 ; Harnack et al., 2009 ; Blanchard et al., 2013).

Dans le cadre d'une prévention primaire et secondaire des RCV, l'ANSES préconise un apport nutritionnel (ANC) en ALA fixé à $1 \%$ de l'apport énergétique total (AET) (ANSES, 2011), ce qui représente une consommation journalière en ALA entre 2 à $3 \mathrm{~g} / \mathrm{j}$. Cependant, les études épidémiologiques ont mis en évidence que, dans les pays occidentaux, l'apport en AGPI $\omega 3$ est insuffisant, en particulier en ce qui concerne l'ALA (Boue et al., 2000). En France, les apports moyens en ALA sont trois fois inférieurs à ceux recommandés (Combe et Boué-Vaysse, 2004 ; Couëdelo, 2014 ; ANSES, 2015). Cette insuffisance d'apport module directement le ratio des précurseurs des séries $\omega 6 / \omega 3$ (LA/ALA), ce qui impacte la biodisponibilité de l'ALA (Combe et Boué-Vaysse, 2004 ; Couëdelo, 2014). En effet, la série des $\omega 6$ partage les mêmes voies enzymatiques de bioconversion et entre en compétition avec celle des $\omega 3$. De ce fait, les précurseurs des deux familles $\omega 3$ et $\omega 6$ doivent être consommés avec un certain équilibre, qui selon les recommandations de 1'ANSES doit tendre vers 5. En réalité, dans les populations occidentales, ce ratio tend plutôt vers des valeurs entre 10 et 14 (Combe et Boue, 2001 ; Astorg et al., 2004 ; Couëdelo et al., 2016). Dans ce contexte, outre l'amélioration des apports en ALA, la prise en compte de la biodisponibilité de l'ALA revêt un champ d'investigation à explorer dans une optique nutritionnelle mais aussi de santé. À ce jour, plusieurs études ont suivi les paramètres susceptibles de moduler l'absorption intestinale et le métabolisme des lipides, avec un intérêt plus prononcé pour les AGPI-LC $\omega 3$. La biodisponibilité de l'ALA est conditionnée par des facteurs physicochimiques, métaboliques et physiologiques (Embleton et Pouton, 1997 ; Armand et al., 1999 ; Singh et al., 2009). Les facteurs moléculaires et supramoléculaires sont un point essentiel à prendre en considération dans les études de biodisponibilité, notamment parce qu'ils impactent la première étape qui conditionne la phase d'absorption, à savoir la lipolyse des éléments lipidiques sous forme d'hydrolysats de lipides ainsi que leur solubilisation micellaire (Michalski et al., 2013).

En ce qui concerne l'ALA, les études sont plus éparses et on s'aperçoit que peu de données sont disponibles quant à l'amélioration de la biodisponibilité de l'ALA. Dans le cadre de nos travaux de recherche, nous sommes attachés à suivre certains paramètres qui permettent d'améliorer la biodisponibilité de l'ALA, en se focalisant sur l'influence de la forme physique et chimique de présentation des lipides ainsi que l'influence de la composition de la matrice alimentaire vis-àvis de la biodisponibilité intestinale et plasmatique de l'ALA. Ces études ont été réalisées sur le modèle rat, modèle validé pour les études d'absorption intestinale des lipides.

\section{Biodisponibilité de l'acide $\alpha$-linolénique-effet de la régio-distribution sur le triglycéride alimentaire}

Les lipides alimentaires sont majoritairement (95\%) présents sous forme de triglycérides (TG) qui ne sont pas absorbables en l'état. Les TG doivent être hydrolysés (lipolyse) par les lipases digestives afin de traverser la barrière intestinale (absorption) et gagner la circulation lymphatique puis plasmatique. Les lipases digestives (lipases gastrique et pancréatique) sont stéréospécifiques des chaînes externes (sn1 et sn3) du TG (Paltauf et Wagner, 1976 ; Mansbach et al., 1985 ; Embleton et Pouton, 1997). Sous leurs actions respectives, un 2-monoacylglycéride (2-MG), issu de la position interne, et deux acides gras libres (AGL), issus des deux positions externes du TG, sont libérés (Fig. 1). Ces 
Tableau 1. Composition et distribution de l'acide alpha-linolénique ( $\mathrm{Ln})$ dans les triglycérides lymphatiques et plasmatiques, après administration de deux structures au cours de la cinétique d'absorption des lipides chez le rat ${ }^{1}$.

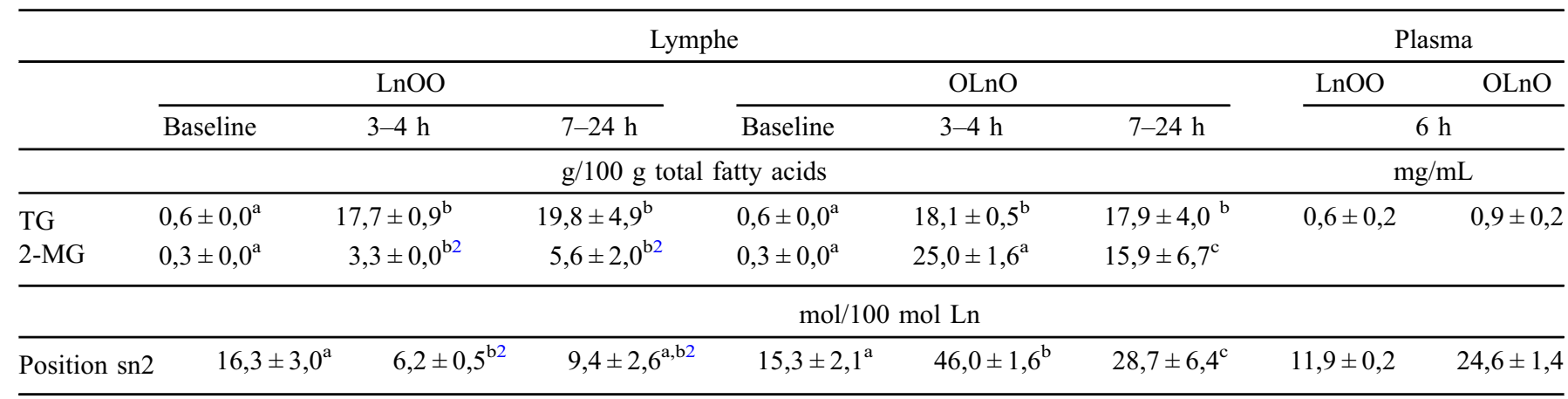

LnOO : acide alpha-linolénique en position sn1/sn3 du triglycéride ; OLnO : acide alpha-linolénique en position sn2 du triglycéride ; TG : triglycéride ; 2-MG : 2-monoacylglycéride.

Les moyennes sans lettre commune pour un même groupe alimentaire diffèrent ; $p<0,05$.

${ }^{1}$ Les valeurs sont représentées par leurs moyennes $\pm \mathrm{SD} ; n=7$ rats.

${ }^{2}$ Différent du groupe OLnO pour un même temps ; $p<0,05$.

produits d'hydrolyse sont alors micellisés et absorbés au niveau de la bordure en brosse de l'entérocyte. Dans l'entérocyte, le 2-MG sert d'amorce pour la synthèse de nouveaux TG. Dans ces conditions, l'ALA distribué en sn2 des TG alimentaires est conservé en cette position dans les TG néosynthétisés et pourrait être ainsi protégé d'une $\beta$-oxydation précoce (Savary et al., 1961 ; Mattson et Volpenhein, 1964 ; El Maghrabi et al., 1978 ; Akesson et al., 1978). Ce pourquoi la position de l'acide gras sur le squelette glycérol et la nature de cet acide gras (longueur de chaîne et degré d'insaturation) modulent aussi leur biodisponibilité (Amate et al., 2001 ; Mu et Høy, 2004 ; Mu et Porsgaard, 2005).

Les données relatives à l'influence de la régio-distribution de l'ALA sur la structure glycéridique s'appuient principalement sur une étude utilisant des huiles naturelles ou en mélange (Boulos et Combe, 2000). Lorsque l'ALA est réparti à hauteur de $31 \%$ en position sn 2 dans l'huile de soja, cette distribution est conservée (29\%) dans les chylomicrons lymphatiques. Si l'on considère l'huile de colza, dont la proportion d'ALA en sn2 est supérieure à celle de soja (58\% de l'ALA en position sn 2 des TG), la distribution de l'ALA en sn2 dans les TG lymphatiques est plus favorable $(40 \%)$ qu'avec l'huile de soja. Toutefois, le maintien de la position de l'ALA sn2 est moindre avec l'huile de colza (75\%) qu'avec l'huile de soja (93\%). Même si la position interne était majoritairement conservée en ce qui concerne l'ALA, il est probable qu'une partie des 2-MG pourrait être hydrolysée par des hydrolases intestinales et conduire à une redistribution de l'ALA sur le squelette glycéridique des TG lymphatiques (Christensen et al., 1995 ; Couëdelo et al., 2012).

L'étude que nous avons menée à partir de TG structurés dont l'ALA était exclusivement greffé soit en position interne soit en position externe (Vaique et al., 2010), nous a permis de suivre le devenir de l'ALA au cours de l'absorption et de la re-synthèse des TG dans l'entérocyte de rat (Couëdelo et al., 2011). Nous avons ainsi pu observer que le niveau d'absorption entérocytaire de l'ALA était similaire, en termes de vitesse et de quantité relative, quelle que soit sa régiodistribution sur le TG alimentaire. Cependant, nous avions observé que, d'une part, la répartition de l'ALA entre la position interne et les positions externes dans les TG lymphatiques était différente selon la molécule considérée et, d'autre part, que la structure glycéridique évoluait au cours de la cinétique d'absorption intestinale des lipides (Tab. 1). Plus précisément, lorsque l'ALA était administré exclusivement en position interne, le maintien de la position représentait $50 \%$ au temps relatif du pic d'absorption des lipides versus $100 \%$ de la molécule structurée administrée (Tab. 1). Deux mécanismes peuvent expliquer l'introduction de l'ALA en position externe des TG synthétisés par l'entérocyte, soit par une isomérisation dans la lumière intestinale, soit une partie de la sn2 monolinolénine absorbée par l'entérocyte a pu être hydrolysée par une lipase avant de servir d'amorce à la synthèse de nouveaux TG (Couëdelo et al., 2012). À l'inverse, lorsque l'ALA était exclusivement greffé en position externe du TG, moins de $10 \%$ de l'ALA était retrouvé en position sn2 des TG lymphatiques. Cette « migration » de position de l'externe vers l'interne peut s'expliquer par la voie de l'acide phosphatidique au détriment de la voie principale des 2-MG.

En effet, sur la fin de cinétique d'absorption des lipides ( 7 à $24 \mathrm{~h}$ post-prandiale), l'ALA en position sn 2 représentait 10 et $30 \%$ lorsqu'il était administré, respectivement, en position externe et interne.

Les mêmes constats ont été obtenus dans le plasma de rat où, six heures après l'administration des lipides, la structure des TG était conservée mais le maintien de la position interne de l'ALA représentait $30 \%$ de la structure initiale (Tab. 1).

Ces études mettent en avant l'intérêt de la position de l'ALA sur le TG alimentaire vis-à-vis des lipases gastriques et intestinales, stéréospécifiques des positions externes du TG. De plus, elles confirment l'impact de la structure glycéridique des TG alimentaires sur celle des TG lymphatiques, en apportant des éléments nouveaux sur le devenir de l'ALA au cours de la resynthèse entérocytaire des $\mathrm{TG}$, en fonction de sa position dans les TG alimentaires. Elles soulignent également l'importance pour l'ALA d'être véhiculé en position interne des TG ingérés, pour son maintien en cette position dans les TG lymphatiques et plasmatiques. Même si la régio-distribution n'est pas 
Tableau 2. Proportions d'acide alpha-linolénique (ALA) dans des huiles végétales ( $\%$ 18:3 $\omega 3$ par rapport aux acides gras totaux) et proportion d'ALA en sn2 par rapport à l'ensemble des positions sn2 (interne) + sn1 et sn3 (externes).

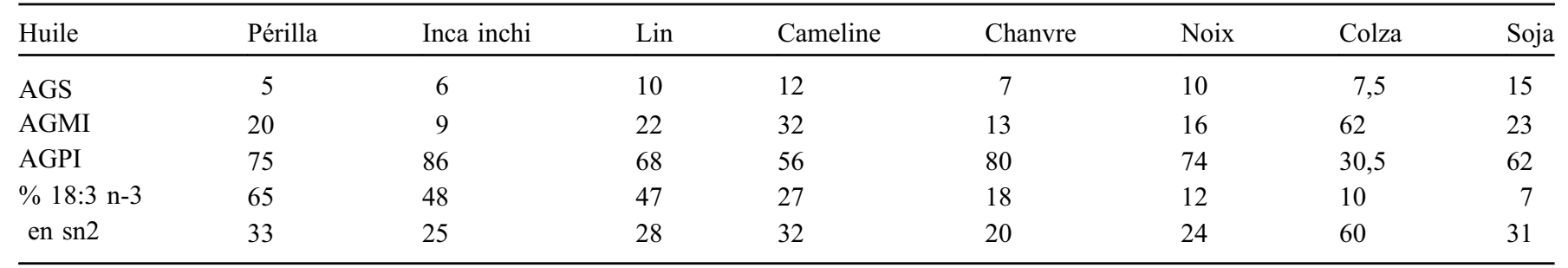

AGS : acides gras saturés ; AGMI : acides gras mono-insaturés ; AGPI : acides gras poly-insaturés.

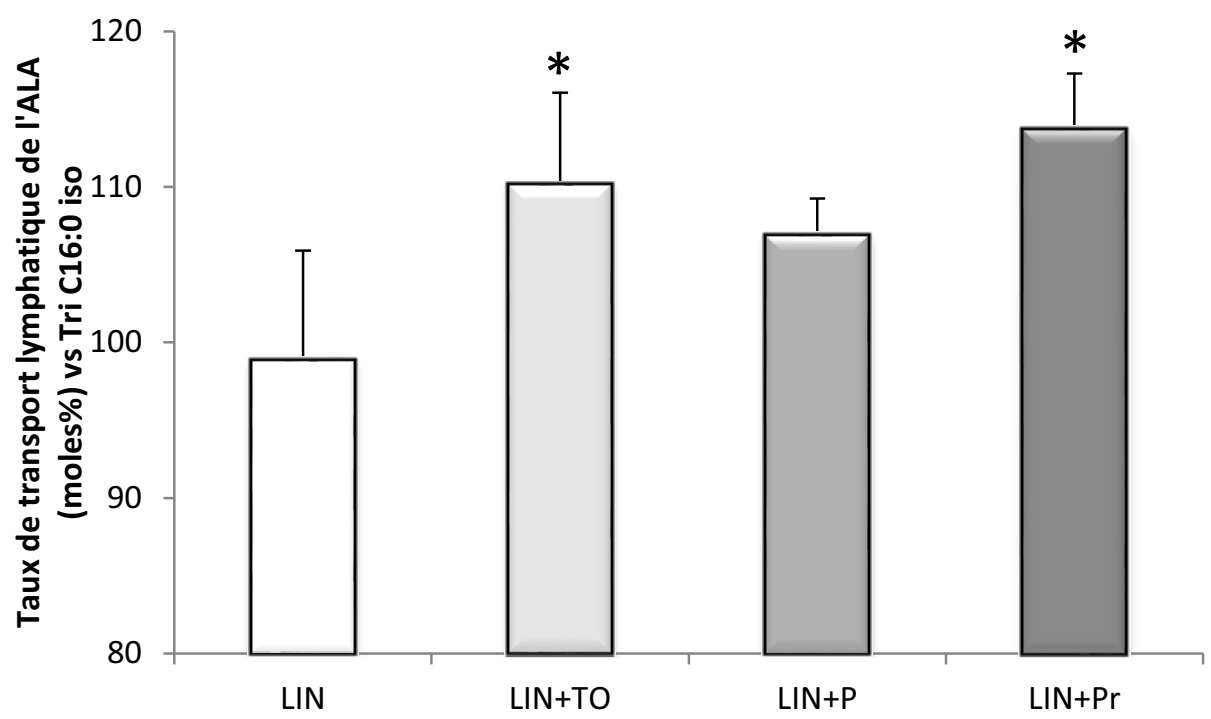

Fig. 2. Taux de passage lymphatique de l'alpha-linolénique (ALA) (mole \%) comparativement à celui de l'acide isopalmitique (référence dont le taux est estimé à $100 \%$ chez des jeunes rats mâles soumis à une dérivation du canal lymphatique mésentérique. Les animaux ( $n=8 /$ groupe) ont reçu par intubation gastrique unique, un bolus de $300 \mathrm{mg}$ de l'une des quatre formules lipidiques (huile de lin soit seule soit combinée à une seconde huile végétale : tournesol oléique [TO], palme [PA] ou pépin de raisin [PR]). Les données marquées d'un astérisque sont significativement différentes $(p<0,05)$, ANOVA monovariée suivie d'un test PLSD de Tuckey.

entièrement conservée, il n'en reste pas moins que la position interne reste à privilégier, pour limiter la $\beta$-oxydation de l'ALA et favoriser son rôle de précurseur des AGPI-LC $\omega 3$ dans les tissus cibles.

\section{Effet de la composition de la matrice lipidique}

Les acides gras sont majoritairement véhiculés dans notre alimentation par des TG. Outre la structure moléculaire, l'influence de la matrice dans laquelle ils sont insérés revêt un intérêt particulier. En fonction de sa composition (nature des acides gras, présence d'antioxydants ou autre nutriments...), la matrice constitue donc un paramètre à prendre en considération dans les études de biodisponibilité.

Les principales sources alimentaires d'ALA sont les huiles linoléniques telles que les huiles de périlla, d'inca inchi, de lin, de colza ou de noix (Tab. 2). Les huiles ont une signature chimique propre et sont caractérisées par leur composition en acides gras, en espèces moléculaires de TG ainsi que par la présence d'autres composés telles que les vitamines. Par conséquent, l'ALA en tant que tel va être soumis aux différents acides gras qui entrent dans la composition de la matrice lipidique.

Dans ce contexte, nous avons évalué l'influence de la nature de certains acides gras vis-à-vis de la biodisponibilité de l'ALA, en combinant l'huile de lin à une seconde huile végétale. L'huile secondaire a été sélectionnée en fonction d'une famille d'acides gras en particulier (le palme pour les AGS, le tournesol oléique [TO] pour les AGMI et le pépin de raisin [PR] pour les AGPI $\omega 6$ ). Le niveau d'absorption intestinale de l'ALA a été suivi en phase aiguë chez des rats porteurs d'une fistulation du canal lymphatique et son devenir métabolique a été évalué au cours d'un régime de huit semaines.

Dans nos conditions expérimentales, nous avons pu observer que le TO et, dans une moindre mesure, le palme permettaient de moduler la biodisponibilité de l'ALA chez le rat adulte. D'une part, le taux d'absorption entérocytaire de l'ALA était amélioré de 11 ou $14 \%$ dès lors que l'huile de lin était associée à de l'huile de $\mathrm{PR}$ ou à du TO, respectivement (Fig. 2). D'autre part, huit semaines de régime avec l'une 
Tableau 3. Composition en acides, alpha-linolénique, eicosapentaénoïque, docosahexaénoïque, dans les différentes fractions lipidiques de foie et de plasma de rats adultes soumis à huit semaines de régime normocalorique-normolipidique. Les lipides sont fournis par l'huile de lin soit seule, soit combinée à une seconde huile végétale (tournesol oléique [TO], palme [PA] ou pépin de raisin [PR] du plasma $[\mu \mathrm{g} / \mathrm{mL}$ ou $\mu \mathrm{g} / \mathrm{g}] ; n=10$ rats/groupe).

\begin{tabular}{llcc}
\hline & \multicolumn{3}{c}{ Plasma $(\mu \mathrm{g} / \mathrm{mL})$} \\
\cline { 2 - 4 } & ALA & EPA & DHA \\
\hline Lin & $108,8^{\mathrm{a}} \pm 39,5$ & $169,0^{\mathrm{a}} \pm 82,7$ & $213,8^{\mathrm{a}} \pm 100,3$ \\
Lin + TO & $267,5^{\mathrm{b}} \pm 90,4$ & $269,0^{\mathrm{b}} \pm 85,9$ & $157,6^{\mathrm{a}} \pm 72,5$ \\
Lin + P & $160,4^{\mathrm{a}} \pm 58,2$ & $180,4^{\mathrm{a}, \mathrm{b}} \pm 51,5$ & $72,5^{\mathrm{a}} \pm 23,4$ \\
Lin + PR & $128,9^{\mathrm{a}} \pm 38,1$ & $150,9^{\mathrm{a}} \pm 29,5$ & $149,0^{\mathrm{a}} \pm 20,7$ \\
\hline & \multicolumn{3}{c}{ TG du foie $(\mu \mathrm{g} / \mathrm{g})$} \\
\cline { 2 - 4 } & EPA & DHA \\
\hline Lin & $397,4^{\mathrm{a}} \pm 138,2$ & $141,6^{\mathrm{a}} \pm 71,6$ & $272,0^{\mathrm{a}} \pm 173,3$ \\
Lin + TO & $168,7^{\mathrm{b}} \pm 45,6$ & $22,5^{\mathrm{b}} \pm 12,4$ & $17,8^{\mathrm{b}} \pm 7,9$ \\
Lin + P & $259,5^{\mathrm{a}} \pm 90,6$ & $36,2^{\mathrm{b}} \pm 12,3$ & $34,6^{\mathrm{b}} \pm 16,6$ \\
Lin + PR & $226,4^{\mathrm{a}} \pm 83,7$ & $26,3^{\mathrm{b}} \pm 10,9$ & $37,8^{\mathrm{b}} \pm 15,0$ \\
\hline
\end{tabular}

\begin{tabular}{lccc}
\hline & \multicolumn{3}{c}{ PL du foie $(\mu \mathrm{g} / \mathrm{g})$} \\
\cline { 2 - 4 } & ALA & EPA & DHA \\
\hline Lin & $78,2^{\mathrm{a}} \pm 20,6$ & $1194,4^{\mathrm{a}} \pm 306,0$ & $272,0^{\mathrm{a}} \pm 173,3$ \\
Lin + TO & $104,9^{\mathrm{a}} \pm 14,4$ & $1454,8^{\mathrm{a}} \pm 125,0$ & $17,8^{\mathrm{b}} \pm 7,9$ \\
Lin + P & $107,0^{\mathrm{a}} \pm 34,6$ & $1471,3^{\mathrm{a}} \pm 241,3$ & $34,6^{\mathrm{b}} \pm 16,6$ \\
Lin + PR & $85,6^{\mathrm{a}} \pm 15,3$ & $1074,5^{\mathrm{a}} \pm 84,6$ & $37,8^{\mathrm{b}} \pm 15,0$ \\
\hline
\end{tabular}

TG : triglycérides ; PL : phospholipides ; ALA : acide alphalinolénique ; EPA : acide eicosapentaénoïque ; DHA : acide docosahexaénoïque. Les données marquées de lettre différente sont significativement différentes $(p<0,05)$ pour un acide gras donné. ANOVA monovariée suivie d'un test PLSD de Tuckey.

des quatre formules conduisaient à des concentrations plasmatiques en ALA, 2,5 fois supérieures en présence de TO et notamment dans les TG du plasma (Tab. 3). Ces résultats suggèrent que la biodisponibilité plasmatique de l'ALA est améliorée en présence de TO. En outre et pour ce régime, les concentrations en EPA étaient également améliorées de près d'un facteur 2 dans les PL plasmatiques, indiquant une amélioration du statut en $\omega 3$ des lipides membranaires.

L'ensemble de ces données expérimentales résultent vraisemblablement d'une meilleure utilisation hépatique de l'ALA chez le rat en présence d'acide oléique (AO), et notamment d'une meilleure bioconversion en EPA (Tab. 3). Les données hépatiques confirmaient cette hypothèse puisque les teneurs en EPA étaient plus importantes dans les PL en combinant l'huile de lin avec du TO ou avec du palme. Ainsi, l'AO apporté par nos régimes TO et palme modulerait la biodisponibilité de l'ALA, notamment vis-à-vis de son absorption intestinale mais aussi de son métabolisme. L'amélioration de transport intestinal de l'ALA observé en présence d'AO permettrait d'expliquer, en partie, les modifications hépatiques et plasmatiques observées.
Tableau 4. Niveaux de lipolyse de l'huile de lin en phase brute ou émulsionnée en conditions in vitro mimant les phases gastrique et duodénale de la digestion.

\begin{tabular}{lcl}
\hline & $\begin{array}{l}\text { Taux de lipolyse } \\
\text { gastrique (\%) } \\
\text { à 29 minutes }\end{array}$ & $\begin{array}{l}\text { Taux de lipolyse } \\
\text { duodénale (\%) } \\
\text { à } 90 \text { minutes }\end{array}$ \\
\hline Huile de lin brute & $12,2 \pm 2,7$ & $39,7 \pm 5,1$ \\
Émulsion caséinate de sodium & $7,3 \pm 2,9$ & $32,6 \pm 4,3$ \\
Émulsion Tween 80 & $2,1 \pm 2,7$ & $28,9 \pm 0,0$ \\
Émulsion lécithine de soja & $16,3 \pm 0,8$ & $50,3 \pm 2,0$ \\
\hline
\end{tabular}

Selon Couëdelo et al., 2015.

\section{Biodisponibilité de l'ALA et effet de l'émulsification des lipides}

Dans notre alimentation occidentale, les lipides sont très souvent formulés et l'émulsification fait partie des procédés qui sont couramment utilisés en IAA. L'émulsification des lipides permet de générer une interface huile/eau qui impacte directement la digestion des lipides. La dispersion des lipides sous forme de gouttelettes lipidiques permet d'augmenter l'interface où s'adsorbent les enzymes lipolytiques et serait de ce fait une étape limitante de la lipolyse (Paltauf et Wagner, 1976 ; Singh et al., 2009 ; McClements et Li, 2010). De précédentes études ont mis en évidence que l'existence de gouttelettes lipidiques préformées lors de l'émulsification accélère la lipolyse gastro-intestinale lors de repas test chez le volontaire sain (Carrière et al., 2000), et induirait une modification de l'accrétion lipidique (Keogh et al., 2011). De plus, les activités catalytiques des lipases sont également dépendantes de la présence d'agents tensioactifs. L'efficacité de la lipolyse dépend donc également de la nature de l'interface (Borgström, 1975 ; Reis et al., 2008 ; Vors et al., 2012, 2013). Or, il existe une variété de surfactants qui sont utilisés en IAA et peu de données ont mis en évidence un impact de leur nature vis-à-vis de l'absorption de l'ALA. Quelques études se sont intéressées à l'impact de la formulation sur le statut plasmatique en ALA. Ces études montrent que la présence de PL et/ou l'émulsification module l'absorption et la biodisponibilité intestinale de l'ALA chez le rat (Nishimukai et al., 2003 ; Nishimukai et Hara, 2007 ; Couëdelo et al., 2011) et la biodisponibilité plasmatique de l'ALA chez l'homme (Garaiova et al., 2007).

Dans ce cadre, nous avons conduit une étude collaborative sur l'impact de la nature de surfactants couramment utilisés en IAA. (caséine, lécithine de soja et Tween 80 ) vis-à-vis de la biodisponibilité de l'ALA (Couëdelo et al., 2015). Dans un premier temps, l'étude in vitro de la lipolyse gastro-intestinale des différentes formules a mis en évidence que le taux de lipolyse de l'huile de lin était amélioré de $50 \%$ avec la lécithine de soja (PL). A contrario, ce taux était diminué de 23 et $37 \%$ en présence de caséine ou de Tween 80 (Tab. 4).

De ces modifications, nous avons pu observer les répercussions in vivo sur une cinétique d'absorption intestinale de l'ALA. Lorsque l'huile de lin est émulsionnée avec la lécithine de soja, la biodisponibilité de l'ALA était améliorée 


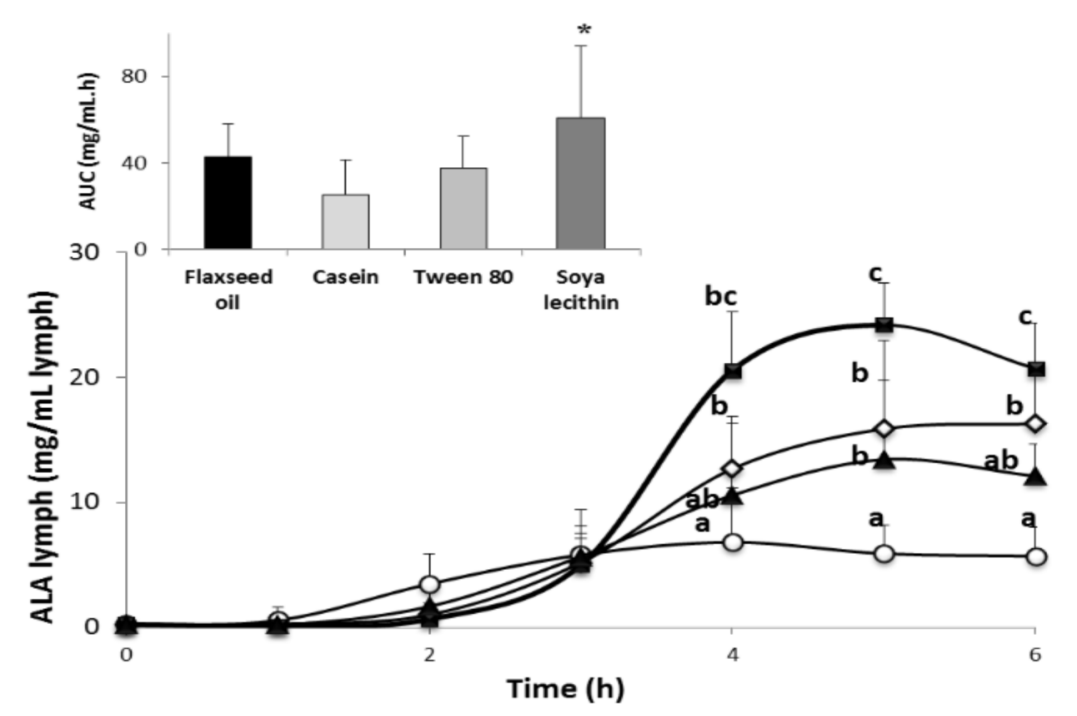

Fig. 3. Évolution de la concentration lymphatique en acide alpha-linolénique (ALA) chez des rats soumis à de l'huile de lin, soit brute ( $\diamond)$ ou émulsionnée avec du caséinate de sodium (O), de la lécithine de soja (ם) ou du Tween $80(\boldsymbol{\Delta})$. Les valeurs représentent la moyenne de huit rats, pour chaque temps de cinétique et pour chaque système lipidique. À partir de $4 \mathrm{~h}$, tous les points diffèrent significativement de la ligne de base. Pour un temps donné, les concentrations sans lettre commune sont significativement différentes ( $p<0,05$; ANOVA suivie du test de Fisher). L'insert représente l'aire sous la courbe (AUC), ${ }^{*} p<0,05$ versus les autres groupes (ANOVA suivi du test post hoc de Fisher test).

Selon Couëdelo et al., 2015.

de $120 \%$, alors qu'en présence de caséine elle était réduite de $42 \%$ (Fig. 3). Une modification de la synthèse des lipoprotéines lymphatiques était par ailleurs observée en fonction de la formule utilisée. Au cours de la cinétique d'absorption des lipides, l'émulsification avec la lécithine de soja induisait une augmentation exponentielle du diamètre moyen des chylomicrons lymphatiques jusqu'à une valeur plateau de $300 \mathrm{~nm}$. À l'inverse, la présence de caséine n'induisait pas cette augmentation exponentielle. L'analyse du nombre de particule de chylomicrons par mesure de l'ApoB48 montre que pour le temps plateau de six heures post-prandiale, la synthèse de particules lipoprotéiques était accrue uniquement avec la lécithine de soja. Dans nos conditions expérimentales, l'émulsification avec des PL de soja conduit, dans un premier temps, à la formation de CM de plus en plus larges, jusqu'à une valeur seuil, à partir de laquelle le diamètre n'augmenterait plus mais le nombre de CM de grande taille serait en revanche augmenté (données non présentées).

Cette étude a permis d'apporter des éléments nouveaux quant au métabolisme des lipoprotéines intestinales. En effet, eu égard aux données, la production des lipoprotéines lymphatiques serait régulée selon deux phases de synthèse ; l'une qualitative où le « cœur » des chylomicrons s'enrichit en TG, générant des CM de plus en plus larges jusqu'à une valeur seuil de $300 \mathrm{~nm}$ (dans nos conditions expérimentales), suivi d'une phase quantitative de stimulation de la synthèse de particules de CM permettant de réguler le flux entrant des lipides. Ces données ont été confirmées par l'étude des gènes codant pour les protéines intervenant dans le compactage des TG (microsomal triglyceride transfer protein [MTTP]), la synthèse des CM (ApoB48) et l'exocytose entérocytaire des CM (Sarlb). L'expression de l'ensemble de ces gènes était significativement améliorée lorsque l'huile de lin était émulsionnée avec de la lécithine de soja comparativement à l'huile de lin brute (Couëdelo et al., 2015).
En outre, des données de la littérature rapportent que de petits $\mathrm{CM}$ seraient plus athérogènes que les grands, en raison d'un taux limité d'hydrolyse des TG des CM (Karpe, 1999) par la lipoprotéine lipase (LPL) (Quarfordt et Goodman, 1966). À l'inverse, des CM plus larges seraient des substrats préférentiels pour la LPL et seraient plus rapidement épurés de la circulation sanguine (Goldberg, 1996). L'action de la LPL a donc pour conséquence une diminution de la lipémie postprandiale, ce qui aurait un effet protecteur vis-à-vis de l'athérosclérose (Martins et al., 1996).

L'étude de la cinétique d'incorporation des lipides dans le compartiment plasmatique a mis en évidence que sur une cinétique post-prandiale de six heures, le diamètre des $\mathrm{CM}$ suivait la même cinétique que celle observée dans la lymphe. Le diamètre moyen des chylomicrons plasmatiques était plus important lorsque l'huile de lin était combinée à la lécithine de soja. La diminution progressive du diamètre des CM observée après le pic d'absorption (deux heures dans le plasma) jusqu'à une valeur basale serait liée à l'action de la LPL. L'activité de la LPL tendait à être plus importante dans le groupe lécithine dénotant que la fixation de la LPL sur des CM plus larges serait facilitée, favorisant l'hydrolyse des $\mathrm{CM}$ en remnants. En outre, suite à l'action de LPL le nombre de petites particules lipoprotéiques était plus important avec la lécithine de soja (Fig. 4).

\section{Conclusion}

Les autorités sanitaires continuent à revoir à la hausse les recommandations actuelles sur l'apport en ALA, qui, selon elles, reste toujours insuffisant dans l'alimentation occidentale. Ainsi, l'engouement de commercialisation des huiles et produits alimentaires riches ou enrichis en $\omega 3$ ne cesse de s'accroître. Cependant, l'ALA est un substrat préférentiel de la 


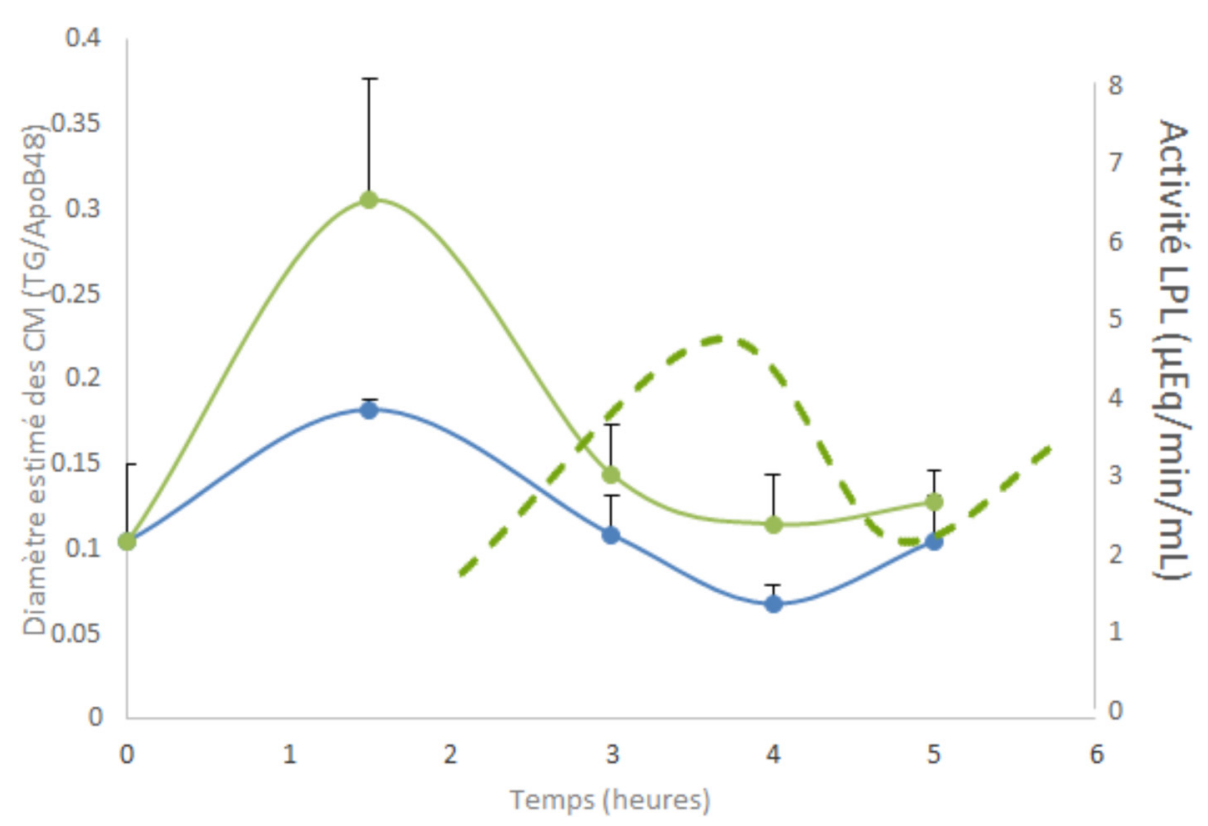

Fig. 4. Cinétiques du diamètre moyen des chylomicrons (CM), estimées par le ratio triglycérides et apoliprotéine B48 (TG/ApoB48), et de l'activité de la lipoprotéine lipase (LPL en $\mu \mathrm{Eq} / \mathrm{min} / \mathrm{mL}$ ) au cours de l'absorption des lipides chez des rats soumis à l'huile de lin brute (bleu) ou soumis à l'huile de lin émulsionnée avec de la lécithine de soja (vert). Les valeurs sont présentées sous la forme moyenne \pm écart-type de 5 rats/groupe.

$\beta$-oxydation et serait ainsi un combustible privilégié pour l'organisme. Augmenter l'apport alimentaire en ALA ne signifie pas pour autant que sa biodisponibilité soit améliorée. Or peu d'études ont été conduites sur les paramètres qui permettent de favoriser l'absorption et le devenir métabolique de l'ALA. La biodisponibilité dépend de plusieurs facteurs qui sont notamment inhérents aux étapes de lipolyse gastrointestinale et de micellisation des acides gras, étapes principales pour leur absorption au niveau de la bordure en brosse des entérocytes.

Quelques études ont pu mettre en avant que la forme chimique d'apport pourrait moduler le métabolisme de l'ALA et que la forme physique (émulsionnée et/ou la présence de PL) améliorerait son absorption entérocytaire chez l'animal et l'homme. Enfin, il a été observé que certains acides gras pourraient améliorer le passage entérocytaire de l'ALA ou au contraire le limiter. Nos études dédiées à l'impact de différents paramètres, pris isolément, sur la biodisponibilité et le métabolisme de l'ALA chez le rat ont permis de mettre en évidence que la régio-distribution de l'ALA sur le TG alimentaire ne module pas son absorption intestinale mais plutôt son devenir métabolique. La position interne permettrait à l'ALA d'être prémuni d'une $\beta$-oxydation et d'être ainsi plus favorablement biodisponible. Nos études confirment que la structure glycéridique des TG alimentaires est partiellement conservée dans les TG lymphatiques et plasmatiques. En outre, ces études ont permis de compléter les informations relatives au devenir de l'ALA au cours de la resynthèse entérocytaire des TG, en fonction de sa position dans les TG alimentaires. Le maintien partiel de la position interne fait suite, pour une partie, à une isomérisation des lipides et certainement à une action d'hydrolyse par des lipases de la position interne (carboxy ester hydrolase [CEH] ou monoacylglycérol lipase $[\mathrm{MGL}])$. Ces études soulignent que même si le maintien de
l'ALA en position interne n'est pas absolu, cette place reste à privilégier pour l'ALA afin de limiter sa $\beta$-oxydation et favoriser ainsi son rôle de précurseur des AGPI $\omega 3$ dans les tissus cibles. De plus amples investigations sont toutefois nécessaires afin de suivre le devenir métabolique de l'ALA selon sa position sur la structure glycéridique dans des tissus d'intérêt, tels que le cœur, le foie et le tissu adipeux.

Outre sa position dans les TG des huiles, l'ALA est soumis à d'autres facteurs qui sont inhérents à la matrice lipidique. Notamment la présence d'autres acides gras peut contribuer à moduler l'absorption et le métabolisme de l'ALA. Nous avons noté que l'association de l'huile de lin avec certaines huiles riches en $\mathrm{AO}$ (notamment le tournesol oléique et, dans une moindre mesure, le palme) permettaient d'améliorer les concentrations plasmatiques et hépatiques en ALA mais aussi en EPA, au terme de huit semaines de régime. Ces données suggéraient une possible spécificité de l'AO vis-à-vis de l'ALA dans son absorption intestinale et son métabolisme hépatique, notamment en termes de bioconversion en EPA. L'amélioration de transport intestinal de l'ALA en présence d'AO permettrait d'expliquer en partie les modifications hépatiques et plasmatiques observées.

En outre, les huiles formulées occupent une place de plus en plus importante dans notre alimentation. Des données de la littérature ont mis en évidence que l'émulsification d'une huile linolénique améliorait l'absorption intestinale de l'ALA en favorisant le compactage des TG dans les chylomicrons lymphatiques (Nishimukai et al., 2003; Nishimukai et Hara, 2007 ; Couëdelo et al., 2011). Nous avons mis en évidence que le procédé d'émulsification avec des PL permettrait d'améliorer la lipolyse gastro-intestinale des TG alimentaires, favorisant la solubilité des acides gras et leur absorption entérocytaire. De plus, l'émulsification avec des PL de lécithine de soja conduisait à la production de CM 
lymphatiques plus gros et plus nombreux, et ce même par rapport à d'autres émulsifiants usuels tels que le Tween $80 \mathrm{ou}$ le caséinate de sodium (Couëdelo et al., 2015).

Nos données ont également permis de faire ressortir que sur une cinétique post-prandiale de six heures, l'émulsification avec des PL de lécithine de soja semble moduler le profil lipoprotéique plasmatique. Les caractéristiques des CM étaient modifiées en termes de taille puis de nombre dès lors que l'huile de lin était émulsionnée avec des PL de soja. Au vu des données de la littérature, il semblerait que dans nos conditions expérimentales, l'émulsification aurait pour conséquence de former, dans un premier temps, des CM de plus en plus larges, jusqu'à une valeur seuil, pour laquelle le diamètre n'augmenterait plus mais à partir de laquelle le nombre de $\mathrm{CM}$ de grande taille serait augmenté. L'activité LPL, alors plus importante dans le groupe émulsion, dénote une fixation facilitée de la LPL sur des CM plus larges et plus nombreux. La clearance des CM, ainsi améliorée, est un facteur favorable pour limiter le risque athérogénique (Martins et al., 1996 ; Mekki et al., 2002).

L'ensemble des données décrites dans ces études confirment l'importance de prendre en compte la structure moléculaire des lipides ainsi que la structure et la composition de la matrice pour moduler la biodisponibilité de l'ALA. Les champs d'investigation vis-à-vis de l'ALA restent encore à explorer dans le but de favoriser la biodisponibilité en fonction de sa forme d'apport tout en limitant la consommation de lipides qui est plus que suffisante dans notre alimentation.

\section{Points importants}

- La structure moléculaire et supramoléculaire des lipides peut moduler la bioaccessibilité et le devenir métabolique de l'ALA. La prise en compte de ces facteurs induit une modification du métabolisme entérocytaire de l'ALA en termes de compactage et de synthèse dans les chylomicrons lymphatiques et plasmatiques.

Déclaration de conflits d'intérêt. Aucun conflit d'intérêt n'est à déclarer.

Remerciements. Leslie Couëdelo et Carole Vaysse remercient l'ANRT, Terres Univia, l'Actia, le FEDER, la région Aquitaine et le GLN pour le financement de certains travaux de recherche réalisés à l'ITERG.

\section{Références}

Akesson B, Gronowitz S, Herslof B, Ohlson R. 1978. Absorption of synthetic, stereochemically defined acylglycerols in the rat. Lipids 13: $338-343$.

Amate L, Gil A, Ramírez M. 2001. Dietary long-chain polyunsaturated fatty acids from different sources affect fat and fatty acid excretions in rats. $J$ Nutr 131: 3216-3221.
ANSES. 2011. Actualisation des apports nutritionnels conseillés pour les acides gras. https://www.anses.fr/fr/system/files/NUT2006 sa0359Ra.pdf.

ANSES 2015. Données de consommation et habitudes alimentaires de l'étude INCA 2. https://www.data.gouv.fr/_uploads/resources/ Table indnut.csv.

Armand $\bar{M}$, Pasquier B, André M, et al. 1999. Digestion and absorption of 2 fat emulsions with different droplet sizes in the human digestive tract. Am J Clin Nutr 70: 1096-1106.

Arterburn LM, Hall EB, Oken H. 2006. Distribution, interconversion, and dose response of n-3 fatty acids in humans. Am J Clin Nutr 83: 1467S-1476S.

Astorg P. 2004. Dietary N-6 and N-3 polyunsaturated fatty acids and prostate cancer risk: a review of epidemiological and experimental evidence. Cancer Causes Control: CCC 15: 367-386.

Bemelmans WJE, Broer J, Feskens EJM, et al. 2002. Effect of an increased intake of alpha-linolenic acid and group nutritional education on cardiovascular risk factors: the Mediterranean Alpha-linolenic Enriched Groningen Dietary Intervention (MARGARIN) study. Am J Clin Nutr 75: 221-227.

Blanchard H, Pédrono F, Boulier-Monthéan N, Catheline D, Rioux V, Legrand P. 2013. Comparative effects of well-balanced diets enriched in $\alpha$-linolenic or linoleic acids on LC-PUFA metabolism in rat tissues. Prostaglandins Leukot Essent Fatty Acids 88: 383-389.

Borgström B. 1975. On the interactions between pancreatic lipase and colipase and the substrate, and the importance of bile salts. J Lipid Res 16: 411-417.

Boue C, Combe N, Entressangles B. 2000. Étude chez une population d'Aquitaine de l'effet des acides gras trans alimentaires sur les lipides plasmatiques et le profil des lipoprotéines. Oléagineux, Corps Gras, Lipides 7: 35-39.

Boulos P, Combe N. 2000. Biodisponibilité de l'acide $\alpha$-linolénique : intérêt d'une huile combinée. Oléagineux, Corps Gras, Lipides 7: 101-104.

Burdge G. 2004. Alpha-linolenic acid metabolism in men and women: nutritional and biological implications. Curr Opin Clin Nutr Metab Care 7: 137-144.

Burdge GC, Wootton SA. 2002. Conversion of alpha-linolenic acid to eicosapentaenoic, docosapentaenoic and docosahexaenoic acids in young women. Br J Nutr 88: 411-420.

Carrière F, Renou C, Lopez V, et al. 2000. The specific activities of human digestive lipases measured from the in vivo and in vitro lipolysis of test meals. Gastroenterology 119: 949-960.

Christensen MS, Müllertz A, Høy CE. 1995. Absorption of triglycerides with defined or random structure by rats with biliary and pancreatic diversion. Lipids 30: 521-526.

Combe N, Boue C. 2001. Apports alimentaires en acides linoléique et alpha-linolénique d'une population d'Aquitaine. OCL 8: 118-121

Combe N, Boué-Vaysse C. 2004. Face aux besoins et à la réalité des consommations, quelles sont les spécificités des différentes sources d'acides gras oméga 3 disponibles ? Oléagineux, Corps Gras, Lipides 11: 103-105.

Couëdelo L. 2014. Evolution of essential fatty acid composition of French breast milk from 1997 to 2014.

Couëdelo L, Boué-Vaysse C, Fonseca L, et al. 2011. Lymphatic absorption of $\alpha$-linolenic acid in rats fed flaxseed oil-based emulsion. Br J Nutr 105: 1026-1035.

Couëdelo L, Vaysse C, Vaique E, et al. 2012. The fraction of $\alpha$-linolenic acid present in the sn-2 position of structured triacylglycerols decreases in lymph chylomicrons and plasma triacylglycerols during the course of lipid absorption in rats. $J$ Nutr 142: 70-75. 
Couëdelo L, Amara S, Lecomte M, et al. 2015. Impact of various emulsifiers on ALA bioavailability and chylomicron synthesis through changes in gastrointestinal lipolysis. Food Funct 6: 1726-1735.

Couëdelo L, Le Guillou C, Pasteau S, Simon N, Tressou J. 2016. Données récentes sur les apports en acides gras des français. Lipid Nutri+ .

El Maghrabi R, Waite M, Rudel LL. 1978. Monoacylglycerol accumulation in low and high density lipoproteins during the hydrolysis of very low density lipoprotein triacylglycerol by lipoprotein lipase. Biochem Biophys Res Commun 81: 82-88.

Embleton JK, Pouton CW. 1997. Structure and function of gastrointestinal lipases. ResearchGate 25: 15-32.

Garaiova I, Guschina IA, Plummer SF, Tang J, Wang D, Plummer NT. 2007. A randomised cross-over trial in healthy adults indicating improved absorption of omega-3 fatty acids by pre-emulsification. Nutr J 6: 4.

Goldberg IJ. 1996. Lipoprotein lipase and lipolysis: central roles in lipoprotein metabolism and atherogenesis. JLipid Res 37: 693-707.

Harnack K, Andersen G, Somoza V. 2009. Quantitation of alphalinolenic acid elongation to eicosapentaenoic and docosahexaenoic acid as affected by the ratio of n6/n3 fatty acids. Nutr Metab 6: 8 .

Hu FB, Stampfer MJ, Manson JE, et al. 1999. Dietary intake of alphalinolenic acid and risk of fatal ischemic heart disease among women. Am J Clin Nutr 69: 890-897.

Karpe F. 1999. Postprandial lipoprotein metabolism and atherosclerosis. J Intern Med 246: 341-355.

Keogh JB, Wooster TJ, Golding M, Day L, Otto B, Clifton PM. 2011. Slowly and rapidly digested fat emulsions are equally satiating but their triglycerides are differentially absorbed and metabolized in humans. J Nutr 141: 809-815.

De Lorgeril M, Renaud S, Mamelle N, et al. 1994. Mediterranean alpha-linolenic acid-rich diet in secondary prevention of coronary heart disease. Lancet 343: 1454-1459.

Mansbach CM, Arnold A, Cox MA. 1985. Factors influencing triacylglycerol delivery into mesenteric lymph. Am J Physiol Gastrointest Liver Physiol 249: G642-G648.

Martins IJ, Mortimer BC, Miller J, Redgrave TG. 1996. Effects of particle size and number on the plasma clearance of chylomicrons and remnants. $J$ Lipid Res 37: 2696-2705.

Mattson FH, Volpenhein RA. 1964. The digestion and absorption of triglycerides. J Biol Chem 239: 2772-2777.

McClements DJ, Li Y. 2010. Structured emulsion-based delivery systems: controlling the digestion and release of lipophilic food components. Adv Colloid Interface Sci 159: 213-228.

Mekki N, Charbonnier M, Borel P, et al. 2002. Butter differs from olive oil and sunflower oil in its effects on postprandial lipemia and triacylglycerol-rich lipoproteins after single mixed meals in healthy young men. $J$ Nutr 132: 3642-3649.
Michalski MC, Genot C, Gayet C, et al. 2013. Multiscale structures of lipids in foods as parameters affecting fatty acid bioavailability and lipid metabolism. Prog Lipid Res 52: 354-373.

$\mathrm{Mu} \mathrm{H}$, Høy CE. 2004. The digestion of dietary triacylglycerols. Prog Lipid Res 43: 105-133.

$\mathrm{Mu} \mathrm{H}$, Porsgaard T. 2005. The metabolism of structured triacylglycerols. Prog Lipid Res 44: 430-448.

Nishimukai M, Hara H. 2007. Soybean phosphatidylcholine-induced enhancement of lymphatic absorption of triglyceride depends on chylomicron formation in rats. Biosci Biotechnol Biochem 71: 1192-1197.

Nishimukai M, Hara H, Aoyama Y. 2003. Enteral administration of soybean lecithin enhanced lymphatic absorption of triacylglycerol in rats. Br J Nutr 90: 565-571.

Paltauf F, Wagner E. 1976. Stereospecificity of lipases. Enzymatic hydrolysis of enantiomeric alkyldiacyl- and dialkylacylglycerols by lipoprotein lipase. Biochim Biophys Acta 431: 359-362.

Quarfordt SH, Goodman DS. 1966. Heterogeneity in the rate of plasma clearance of chylomicrons of different size. Biochim Biophys Acta 116: 382-385.

Reis PM, Raab TW, Chuat JY, et al. 2008. Influence of surfactants on lipase fat digestion in a model gastro-intestinal system. Food Biophys 3: 370-381.

Riediger ND, Othman RA, Suh M, Moghadasian MH. 2009. A systemic review of the roles of n-3 fatty acids in health and disease. J Am Diet Assoc 109: 668-679.

Savary P, Constantin MJ, Desnuelle P. 1961. On the structure of the triglycerides of the lymphatic chylomicrons in the rat. Biochim Biophys Acta 48: 562-571.

Simopoulos AP. 2008. The importance of the omega-6/omega-3 fatty acid ratio in cardiovascular disease and other chronic diseases. Exp Biol Med (Maywood, NJ) 233: 674-688.

Singh H, Ye A, Horne D. 2009. Structuring food emulsions in the gastrointestinal tract to modify lipid digestion. ResearchGate 48: 92-100.

Vaique E, Guy A, Couedelo L, et al. 2010. Rapid access to structured triacylglycerols acylated with n-3 polyunsaturated fatty acids for nutritional applications. Tetrahedron 66: 8872-8879.

Vors C, Capolino P, Guérin C, et al. 2012. Coupling in vitro gastrointestinal lipolysis and Caco-2 cell cultures for testing the absorption of different food emulsions. Food Funct 3: 537-546.

Vors C, Pineau G, Gabert L, et al. 2013. Modulating absorption and postprandial handling of dietary fatty acids by structuring fat in the meal: a randomized crossover clinical trial. Am J Clin Nutr 97: 23-36.

Zhao G, Etherton TD, Martin KR, West SG, Gillies PJ, Kris-Etherton PM. 2004. Dietary alpha-linolenic acid reduces inflammatory and lipid cardiovascular risk factors in hypercholesterolemic men and women. J Nutr 134: 2991-2997. 